\title{
Perspectiva cualitativa en la construcción del conocimiento didáctico del contenido del profesorado de Educación Física Qualitative perspective in the construction of didactic content knowledge in Physical Education teachers \\ *Aquiles Alejandro Almonacid-Fierro, **Eugenio Merellano-Navarro, ***Sebastián Feu Molina, Manuel Vizuete Carrizosa, ** Rosa Orellana Fernández \\ *Universidad Católica del Maule (Chile), **Universidad Autónoma de Chile (Chile), ***Universidad de Extremadura (España)
}

\begin{abstract}
Resumen. La presente investigación tiene como propósito describir el discurso y la práctica docente de aula de los profesores de Educación Física en relación con el Conocimiento Didáctico del Contenido (CDC). La construcción del conocimiento didáctico y consecuentemente pedagógico se genera desde las prácticas escolares, produciéndose un aprendizaje de conocimiento articulado y situado en la relación entre teoría y práctica, que comprende aspectos didácticos, pedagógicos, disciplinares, profesionales y prácticos de la profesión docente. En este sentido, la investigación se planteó como objetivo comprender el proceso de construcción y las fuentes del conocimiento didáctico del contenido en el profesorado de Educación Física de la región del Maule - Chile. La metodología utilizada es de carácter cualitativa, toda vez que se sustenta en el paradigma interpretativo-comprensivo que busca la comprensión de fenómenos complejos. En términos de diseño, se realizaron seis entrevistas semiestructuradas a profesores de Educación Física que trabajan en la Región del Maule, Chile. El proceso de codificación, categorización e interpretación permitió concluir que poseer un conocimiento de carácter disciplinar por parte del profesor, no garantiza aprendizajes de calidad para los estudiantes que participan del aula de Educación Física, puesto que se requiere de un conocimiento pedagógico general, que permita al profesor planear, organizar y ejecutar situaciones de enseñanza-aprendizaje de calidad.
\end{abstract}

Palabras clave: Docente, conocimiento, pedagogía, educación física.

\begin{abstract}
The aim of this research is to describe the discourse and the classroom teaching approach of Physical Education teachers in relation to the Didactic Content Knowledge (CDC). The construction of didactic and consequently pedagogical knowledge is generated from school practices, producing an articulated learning knowledge and located in the relationship between theory and practice, which includes didactic, pedagogical, disciplinary, professional, and practical aspects of the teaching profession. In this sense, the objective of this research was to understand the construction process and the sources of CDC in Physical Education teachers from the Maule region in Chile. The methodology used is qualitative, since it is based on the interpretive-comprehensive paradigm that seeks to understand complex phenomena. In terms of design, six semi-structured interviews were conducted with Physical Education teachers working in the Maule Region, Chile. The process of coding, categorization, and interpretation allowed us to conclude that even if teachers have disciplinary knowledge that does not guarantee quality learning for students who participate in the Physical Education classroom, since a general pedagogical knowledge is required, which allows teachers to plan, organize, and execute quality teaching-learning situations.
\end{abstract}

Keys Words: Teacher, knowledge, pedagogy, physical education.

\section{Introducción}

Estudiar el Conocimiento Didáctico del Contenido (CDC) de los profesores significa adentrarse en cuestiones claves de la profesión docente. Esto implica tensionar el ethos profesional y contribuir al tránsito desde una posición tradicional del profesor como transmisor del conocimiento, a una lectura en donde el profesional que es capaz de aplicar saberes pedagógicos, disciplinares y didácticos en el aula, desde una concepción reflexiva, critica, integradora y compleja del fenómeno humano y educativo. Este reflexionar crítico favorece el descubrimiento de nuevas formas para aprender y enseñar (Fernandez, 2015; Gitomer \& Zisk, 2015; Jimenez, Rossi, \& Gaitán, 2017; Schön, 1998).

El CDC representa la combinación de conocimientos formales o proposicionales que el profesor domina, no obstante, sólo se constituye en la medida que una situación real de enseñanza tiene lugar. $\mathrm{Al}$ ser reelaborado por el profesor en la acción de enseñanza, el CDC incorpora otras características y asume otro formato, vale decir, una nueva estructuración práctica con importantes repercusiones para el aprendizaje de los estudiantes y para la propia reconfiguración del pro-

Fecha recepción: 22-11-18. Fecha de aceptación: 26-05-19 Aquiles Alejandro Almonacid-Fierro aalmonacid@ucm.cl ceso de enseñanza del maestro (Vázquez-Bernal \& JiménezPérez, 2019).

El profesor es un profesional que elabora conocimientos importantes a partir de la confrontación de situaciones problemáticas que surgen desde la práctica cotidiana de aula que no se resuelven con el saber científico ya elaborado. Por otro lado, la experiencia profesional del profesor y la forma particular en cómo este interpreta y utiliza esa experiencia, contribuyen al desarrollo de un tipo de conocimiento procesual muy útil para su intervención en el aula. La experiencia, en este sentido, en un elemento crucial en el proceso de elaboración del conocimiento didáctico del contenido. En este sentido, Cañadas, Santos-Pastor, \& Castejón (2019) plantean que «un dominio adecuado sobre qué y cómo enseñar la materia, supone que el docente deberá diseñar las tareas con las que pretende que el alumnado aprenda el contenido, ponga en práctica las actividades según diferentes técnicas, estilos y estrategias de enseñanza» (p. 284), cuestiones que emergen desde la relacionalidad del aula.

De acuerdo con lo señalado precedentemente, los programas de formación inicial de profesores de Educación Física en Chile deben considerar especialmente esta cuestión, especificando cuál es el papel que asume la experiencia práctica en cada momento de formación. Si bien varios cursos de formación de profesores en el área en Chile se han estructurado de modo tal que la experiencia práctica en el aula escolar 
se realice al final de la formación, la experiencia no aparece como una oportunidad de formación de nuevas competencias, sino más bien como un despliegue de competencias ya aprendidas. En consecuencia, nos interrogamos sobre la necesidad de que los programas formativos consideren en todo momento la planeación y ejecución de situaciones de enseñanza en que se integren permanentemente, el contenido, la pedagogía y el contexto (Amade-Escot, 2000; Marcon, Nascimento, \& Graça, 2007; Nascimiento, 2006; Pedroso, 1996; Ramos, dos Santos Graça, \& do Nascimiento, 2008). Resulta, no obstante, relevante identificar las categorías generales de conocimiento que poseen los maestros, tales como: sus creencias, el conocimiento de la materia, el conocimiento de los estudiantes, el conocimiento de la pedagogía, del currículo, de la evaluación, del contexto y otras categorías del proceso enseñanza-aprendizaje. Esto permitiría conocer y comprender el proceso de construcción y las fuentes. Esta interrogante nos permite plantear dos hipótesis de investigación. La primera es que el constructo CDC es escasamente conocido por el profesorado de educación física, y no existe un instrumento que permita valorarlo en todas sus dimensiones. Una segunda hipótesis es que los contenidos enseñados en el área de Educación Física están en relación con habilidades motrices básicas, juegos deportivos y deportes, ejercicio físico y salud, de tal manera que las manifestaciones motoras asociadas a la cultura juvenil, la expresión corporal y/o a las actividades al aire libre, se encuentran reducidas a una mínima expresión.

La investigación del CDC se ha desarrollado desde la década del 80 a través de diferentes programas de investigación (Barnett \& Hodson, 2001; Bolivar, 1993; Fenstermacher, 1994; Shulman, 1986; Shulman, 1987). En la disciplina de Educación Física, la mayor parte de los estudios del CDC utiliza el análisis de contenido en el marco de la teoría fundamentada, teniendo como telón de fondo el paradigma interpretativocomprensivo, cuestión que lleva a los investigadores a plantear los siguientes hallazgos:

Los profesores de EF poseen un enfoque basado preferentemente en el constructivismo.

El conocimiento de los profesores de Educación Física expertos está altamente organizado e integrado, cuestión que favorece el aprendizaje por parte de los estudiantes.

Lo anterior en contraste con los profesores novatos, que tienen problemas para conectar el contenido de la lección en contextos más amplios.

Los profesores hacen la conexión para ayudar a los niños a entender el significado del contenido de la lección como parte de un todo mayor, es decir, el deporte, la actividad física o su vida fuera de la escuela. Por lo tanto, los maestros de EF presentan contenidos en un contexto que otorga significado al contenido y consecuentemente fortalece el CDC.

- Otro hallazgo sugiere que es un error de la formación de profesores de EF enfatizar en una modalidad de la formación, ya sea disciplinaria o pedagógica. Más bien, lo que se necesita es una amalgama lógica de las dos formas de conocimiento, vale decir la imbricación y/o conjunción compleja de un conocimiento disciplinar y pedagógico a la vez (Almonacid-Fierro, Feu, \& Carrizosa, 2018; Capel, Hayes, Katene, \& Velija, 2009; Castejon \& Giménez, 2017; Hunuk,
Ince, \& Tannehill, 2013; MacPhail, Tannehill, \& Karp, 2013; Mancha, 2012; Marcon, Graça, \& Nascimento, 2012; Stran \& Curtner-Smith, 2010; Ward, Kim, Ko, \& Li, 2015).

El conocimiento del contenido asume una importancia relevante en la formación inicial de futuros profesores de Educación Física constituyéndose un prerrequisito imprescindible para el desarrollo adecuado del conocimiento pedagógico del contenido ya que de ésta deriva el propio contenido que será enseñado a sus alumnos. En este contexto, el objetivo de este estudio es describir y categorizar el discurso y la práctica docente de aula de los profesores de Educación Física en relación con el conocimiento didáctico del contenido en el profesorado de Educación Física de la región del Maule, Chile

\section{Material y método de investigación}

La presente investigación es un estudio de tipo interpretativo en el que se pretende indagar sobre las concepciones referidas a CDC de los profesores de Educación Física de la región del Maule. Nuestro diseño de investigación se enmarca en una lógica descriptiva de propiedades, características y perfiles de personas, grupos, comunidades, procesos, objetos o cualquier otro fenómeno que se someta a un análisis. Se adopta una metodología de tipo cualitativa, con el propósito de acercarnos a la comprensión subjetiva que tienen los sujetos en relación con el CDC y la manera en cómo ellos significan, representan y de alguna manera dan sentido a los saberes aprendidos en su formación inicial y que luego transmiten en su ejercicio profesional (Canales, 2006; Delgado \& Gutiérrez, 1999; Flores, 2009; Gibbs, 2012; Rodríguez, Gil, \& García, 1996; Taylor \& Bogdan, 1987).

La investigación se estructuro en seis fases:

$\varnothing$ Fase 1: Definición de la muestra, la que se realizó a través del muestreo teórico.

Ø Fase 2: Validación del guion de entrevista por un panel de cinco jueces, académicos investigadores de universidades chilenas y españolas.

$\varnothing$ Fase 3: Aplicación de las entrevistas. Esta fase se realizó durante los meses de noviembre y diciembre del año 2016, tuvieron una duración de 60 minutos.

Ø Fase 4: Transcripción y análisis: una vez transcritas las entrevistas se sometieron a análisis de contenido.

$\varnothing$ Fase 5: Codificación y categorización: se realizó una codificación abierta, cuestión que hizo emerger de los datos un gran abanico de códigos, y seguidamente posibilitó ir integrando los códigos en unidades mayores denominadas categorías secundarias, para luego agruparlas en categorías primarias, estableciendo las correspondientes relaciones con la pregunta de investigación.

$\varnothing$ Fase 6: Interpretación: una vez que se sistematizaron los datos en una matriz, se procedió al proceso de interpretación en base a la Teoría Fundamentada (Glaser \& Strauss, 2017).

\section{Resultados}

La tabla 1 muestra los atributos de los profesores que participaron en la entrevista, evidenciándose que el 50\% de los entrevistados realizó sus estudios de pregrado en la 
Universidad Católica del Maule, cuestión comprensible debido a que la institución tiene 50 años de presencia en la región y en particular en la formación de profesores de esta disciplina.

\begin{tabular}{|c|c|c|c|c|}
\hline $\begin{array}{c}\mathrm{N}^{\circ} \\
\text { Entrevista }\end{array}$ & Institución ES & Año egreso & Lugar de trabajo & Años de docencia \\
\hline 1 & $\begin{array}{l}\text { Universidad Católica } \\
\text { del Maule }\end{array}$ & 2010 & $\begin{array}{l}\text { Liceo Claudina Urrutia- } \\
\text { Cauquenes }\end{array}$ & 6 años \\
\hline 2 & $\begin{array}{c}\text { Pontificia Universidad } \\
\text { Católica de Chile }\end{array}$ & 1990 & Colegio Piaget- Talca & 22 años \\
\hline 3 & Universidad del Mar & 2011 & $\begin{array}{l}\text { Escuela San Valentín - } \\
\text { Curicó }\end{array}$ & 5 años \\
\hline 4 & $\begin{array}{c}\text { Universidad } \\
\text { Autónoma de Chile }\end{array}$ & 2008 & $\begin{array}{c}\text { Colegio Camilo } \\
\text { Henríquez - Talca } \\
\end{array}$ & 6 años \\
\hline 5 & $\begin{array}{l}\text { Universidad Católica } \\
\text { del Maule }\end{array}$ & 2009 & $\begin{array}{l}\text { Colegio Manzo de } \\
\text { Velasco-Cauquenes }\end{array}$ & 7 años \\
\hline 6 & $\begin{array}{c}\text { Universidad } \\
\text { Autónoma de Chile }\end{array}$ & 2009 & $\begin{array}{c}\text { Colegio Marta Brunet - } \\
\text { Linares }\end{array}$ & 7 años \\
\hline
\end{tabular}

Los entrevistados presentan en promedio 6 años de experiencia docente, excepto un caso que llega a los 22 años. Lo anterior, nos permite deducir que son profesores que cuentan con experiencia en el sistema escolar y por tanto pueden realizar un análisis crítico-reflexivo de su práctica pedagógica, específicamente de los conocimientos requeridos en su área profesional. En términos de representatividad regional, la muestra considera profesores de cada una de las provincias que integran la región del Maule.

Se aplicaron seis entrevistas siguiendo las recomendaciones de Flores (2009), Kvale (2011) y Taylor y Bogdan (1987). Las entrevistas se trabajaron bajo la perspectiva teórica de la teoría fundamentada (Coffey \& Atkinson, 2003; Gibbs, 2012; Strauss \& Corbin, 2002).

En la Tabla 2 se presentan las categorías primarias con su correspondiente descriptor sobre la manera en cómo se comprende el fenómeno y las diferentes dimensiones del CDC. Una vez definidas las categorías previas se comienza el proceso de codificación. En este contexto, se inició el proceso de simplificación o reducción de los datos, para seguidamente asignar los primeros conceptos, que en el marco de esta investigación se denominan categorías secundarias (Coffey \& Atkinson, 2003). A este proceso Strauss y Corbin (2002) lo denominan codificación abierta, por tanto, las categorías primarias representan esencialmente fenómenos que provienen de las subcategorías (categorías secundarias en este caso), que le permiten dar claridad adicional y especificidad a los datos.

\section{Interpretación categorías secundarias}

Para efectos de este artículo, se analizan todas las cate- gorías primarias de cada categoría previa. La identidad de los sujetos entrevistados ha sido protegida según las consideraciones éticas definidas para este estudio. Los nombres que aquí aparecen son simbólicos y se utilizaron como código del relato.

Categoría Previa Conocimiento del Contenido Específico - Categoría Primaria Área de Conocimiento Específico - Categoría Secundaria Ámbito Pedagógico:

Los informantes expresan que un profesor debe poseer un soporte pedagógico al momento de desplegar el proceso enseñanza-aprendizaje.

«Las psicologías que tenemos dentro de la carrera, que tenemos tres psicologías, que son para entender cómo piensa el niño, cómo se va desarrollando, cómo podemos enseñar los ejercicios, los contenidos; ya que si no tenemos claro cuáles son las formas de aprendizaje que tienen los niños es muy difícil hablar en un lenguaje y que sea entendible para ellos. Entonces, es importante manejar una psicología sobre cómo tratar con los niños ya que todos son sujetos individuales». Entrevista Juan [Cobertura 0,81\%]

El dominio del ámbito pedagógico y, específicamente, los aspectos referidos a la psicología del desarrollo y el aprendizaje permiten al profesor ir desplegando los diferentes objetivos del área en los diversos grupos etarios donde le corresponde actuar desde primaria hasta la secundaria. Este aspecto es relevante cuando el profesor de la disciplina Educación Física puede ejercer su labor docente tanto en enseñanza primaria como secundaria y entonces atender a alumnos de entre 6 años y 17 años (Ministerio de Educación de Chile, 2013).

Categoría Previa Conocimiento del Contenido Específico - Categoría Primaria Área de Conocimiento Específico - Categoría Secundaria Dominio Disciplinar.

Los profesores investigados expresan que el dominio disciplinar es un elemento sustantivo para el desarrollo de su docencia.

«Dentro de la formación, todo lo que tenga que ver con el contenido de educación física y movimiento, psicomotricidad, pasar todos los deportes. La base teórica que debemos tener en lo que son los conocimientos dentro de los contenidos de la educación física... uno debiese manejarlo a cabalidad o en un $80 \%$, ya que hoy en día hay alumnos inquietos que realizan muchas preguntas y el pro-

\begin{tabular}{|c|c|c|c|c|c|c|c|}
\hline Mega categoría & $\begin{array}{c}\text { Categoría } \\
\text { previa }\end{array}$ & Categoría primaria & Descriptor & Categorías secundarias & Referencias & $\begin{array}{l}\text { \% Categoría } \\
\text { Previa } \\
\end{array}$ & $\%$ Tota \\
\hline \multirow{18}{*}{$\begin{array}{l}\text { Conocimiento didáctico } \\
\text { del contenido en } \\
\text { educación física }\end{array}$} & \multirow{10}{*}{$\begin{array}{l}\text { Conocimiento } \\
\text { del contenido } \\
\text { especifico }\end{array}$} & \multirow{3}{*}{$\begin{array}{l}\text { Área de conocimiento } \\
\text { especifico }\end{array}$} & \multirow{3}{*}{$\begin{array}{l}\text { Esta categoría considera las áreas de conocimiento- } \\
\text { que maneja el profesor de Educación Física. }\end{array}$} & Ámbito pedagógico & 2 & 4,8 & 2,78 \\
\hline & & & & Dominio disciplinar & 5 & 11,9 & 6,94 \\
\hline & & & & Hab. motrices básicas & 3 & 7,1 & 4,17 \\
\hline & & Contenido de la Educación & Agrupa lo que dice relación con los contenidos de la & Contenido contextualizado & 4 & 9,5 & 5,56 \\
\hline & & Física & EFI escolar. & Contenidos más utilizados en $\mathrm{EF}$ & 4 & 9,5 & 5,56 \\
\hline & & Proceso evaluativo & $\begin{array}{l}\text { Los aspectos que dicen relación con el proceso } \\
\text { evaluativo en el aula de EFI. }\end{array}$ & Evaluación de proceso & 4 & 9,5 & 5,56 \\
\hline & & \multirow{4}{*}{ Rol de profesor } & \multirow{4}{*}{$\begin{array}{l}\text { Categoría que alude a los diferentes roles que asume } \\
\text { el profesor en el proceso enseñanza-aprendizaje. }\end{array}$} & Empatía pedagógica & 7 & 16,7 & 9,72 \\
\hline & & & & Motivación para la clase & 5 & 11,9 & 6,94 \\
\hline & & & & Profesor lúdico & 3 & 7,1 & 4,17 \\
\hline & & & & Profesor modelo & 5 & 11,9 & 6,94 \\
\hline & \multirow{8}{*}{$\begin{array}{l}\text { Conocimiento } \\
\text { pedagógico } \\
\text { general }\end{array}$} & \multirow{2}{*}{ Déficits en la formación } & La categoría da cuenta de aquellas materias que no se & En lo administrativo & 7 & 20,0 & 9,72 \\
\hline & & & abordaron suficientemente en la FID. & Área Pedagógica & 5 & 14,3 & 6,94 \\
\hline & & \multirow{2}{*}{ Pedagogía del profesor } & Los elementos pedagógicos con los que el profesor & Actitud indagativa & 2 & 5,7 & 2,78 \\
\hline & & & despliega su clase. & Actitudes transversales & 4 & 11,4 & 5,56 \\
\hline & & \multirow{2}{*}{ Planificación de la enseñanza } & El abordaje de la sesión requiere un proceso de & Planificación contextualizada & 4 & 11,4 & 5,56 \\
\hline & & & planificación con ciertas características. & Planificación flexible & 6 & 17,1 & 8,33 \\
\hline & & \multirow{2}{*}{ Reflexión sobre la practica } & Categoría que permite comprender como el proceso & Instancia reflexiva & 4 & 11,4 & 5,56 \\
\hline & & & mejora su práctica a través de un ejercicio reflexivo. & Retroalimentar para aprender & 3 & 8,6 & 4,17 \\
\hline
\end{tabular}


fesor de educación física o el profesor en general debiese manejar las respuestas de aquellas inquietudes». Entrevista Marco [Cobertura 1,08\%]

Los sujetos investigados hacen referencia a los contenidos más comúnmente usados en el área Educación Física, haciendo mención específicamente a la psicomotricidad y a los deportes. Lo anterior daría cuenta de un modelo formativo más bien tradicional o «técnico» de acuerdo con lo que expone García (1997). En el referido modelo, el ideal educativo en la disciplina de Educación Física está orientado hacia la formación de un ser humano productivo, prestando especial atención a los elementos de carácter técnico. Lo anterior se ve reflejado en una didáctica que privilegia el individualismo, la marca y el modelamiento de gestos motores provenientes, fundamentalmente del deporte. Otros autores (Benito-Capa, Portela-Lozano, \& Rodríguez-Jiménez, 2006; Jaramillo, Murcia, \& Portela, 2005; Kirk, 2001; Moreno, Campos, Almonacid, \& Vargas, 2013; López, Monjas y Pérez, 2003), dan cuenta de la mirada más bien tecnicista que predomina en los currículos de formación de profesores de Educación Física, puesto que el conocer y dominar los deportes representa un porcentaje importante de la formación profesional, y la cuestión más sustantiva al respecto, es que los métodos de enseñanza y los procesos evaluativos se orientan a la performance técnica, vale decir a que el estudiante primero y el profesor después, demuestre una competencia elevada en deportes individuales o de conjunto. Todo ello se traduce posteriormente en las aulas de escuelas y liceos, en sesiones de desarrollo deportivo en los que privilegia la técnica y el rendimiento, separando a los más hábiles de los menos hábiles.

Categoría Previa Conocimiento del Contenido Específico - Categoría Primaria Área de Conocimiento Específico - Categoría Secundaria Habilidades Motrices Básicas.

Los profesores entrevistados consideran importante el trabajo sobre las habilidades motrices básicas, pues es considerado un pilar fundamental con el que seguidamente construyen los juegos predeportivos.

«Trabajo los fundamentos básicos las habilidades motrices básicas de cada niño, equilibrio, salto. Las habilidades básicas porque eso nos va a llevar después a trabajar los juegos predeportivos». Entrevista Silvio [Cobertura 3,73\%]

El desarrollo de las habilidades motrices básicas es una tarea que se aborda desde la primera infancia, con el trabajo que desarrollan preferentemente la educadora de párvulos con niños de 4 y 5 años y seguidamente los profesores de educación básica generalista, y hoy, en mayor medida, los profesores de educación física, con niños de 6 a 9 años. En este contexto, se observa que el discurso de los participantes se encuentra en sintonía con los lineamientos curriculares del Ministerio de Educación establecidos en las Bases Curriculares.

Categoría Previa Conocimiento del Contenido Específico - Categoría Primaria Contenido de la Educación Física- Categoría Secundaria - Contenidos Contextualizados
Los profesores hacen referencia al desarrollo de los contenidos durante la sesión y la posibilidad que tiene el docente de adecuar aquellos contenidos planificados para la unidad de acuerdo con el contexto en que se encuentra inserto.

«Hay que enseñarle al niño ¡cuál es una buena técnica! ¡cómo se lanza bien! A lo mejor, hay que identificar que al niño le falta un poquito más de coordinación o le falta fuerza o tiene un patrón de movimiento no adquirido y hay que corregir eso. Enseñar eso que para el niño pueda lograr ese objetivo o ver bien los contenidos que se están trabajando, ver el promedio del curso. A lo mejor, los movimientos son muy complejos para la edad del desarrollo muscular que tienen esos niños y hay adecuarlos a la realidad que tiene el curso para no generar la frustración en ellos. Entrevista Juan [Cobertura 3,34\%]

Los relatos de los profesores entrevistados dan cuentan de la manera en cómo ellos son capaces de considerar la edad de los niños, su desarrollo motor, así como también las condiciones de infraestructura y equipamiento con las que cuenta el docente para el desarrollo de los contenidos planificados. Lo anterior permite comprender que el conocimiento del profesor necesita ser adaptado a la especificidad del contexto de enseñanza-aprendizaje, teniendo especial consideración a las características y circunstancias del ambiente escolar, las condiciones del aula y fundamentalmente las peculiaridades de determinado grupo de alumnos.

Categoría Previa Conocimiento del Contenido Específico - Categoría Primaria Contenido de la Educación Física-Categoría Secundaria - Contenidos más utilizados en Educación Física (EF).

La categoría dice relación con los contenidos mayormente utilizados por los profesores de Educación Física en su ejercicio docente, en los diferentes establecimientos educacionales donde trabajan.

«Una de las actividades que tiene más relevancia en el establecimiento de los años es la práctica de actividades motrices al aire libre. Considero que son prácticas que son mucho más abiertas en donde los jóvenes y los niños pueden realizar actividades que no estén direccionadas, que pueden ampliar un poco su mente con respecto al medio natural». Entrevista Miguel [Cobertura 3,18\%]

De acuerdo con lo expuesto por los profesores informantes los contenidos mayormente utilizados en el área de la Educación Física son los referidos a: área corpóreo-motriz; juegos predeportivos; deportes; actividades en contacto con la naturaleza; vida activa y saludable, seguridad. Las bases curriculares actuales consideran que el área Educación Física y Salud al ser una asignatura que se enmarca en el proceso de formación integral del ser humano, favorece la práctica regular de actividad física en los estudiantes y de esta manera ellos pueden desarrollar habilidades motrices, actitudes inclinadas al juego limpio, el liderazgo y el autocuidado. Cuestión que finalmente redundará en la posibilidad de adquirir un estilo de vida activo y saludable, asociado a múltiples beneficios individuales y sociales preconizados en el curriculum nacional (Ministerio de Educación de Chile, 2013).

Categoría Previa Conocimiento del Contenido Específico - Categoría Primaria Proceso Evaluativo-Categoría Secundaria - Evaluación de Proceso. 
«Conocer a los niños, qué objetivo es el que busco, qué herramientas voy a utilizar para llegar a ese objetivo; teniendo como eje fundamental, la evaluación que voy a hacer para nuevamente retroalimentar y volver a superar las debilidades que tuve, en esa evaluación que fue realizada por los alumnos». Entrevista Joaquín [Cobertura 1,27\%]

Los relatos dan cuentan sobre cómo los profesores conciben la acción de evaluar, con la idea de ir mejorando y retroalimentando el proceso de enseñanza y aprendizaje. Lo anterior permite inferir que la evaluación supera la instancia de una calificación, y se acerca más bien a un momento de aprendizaje, como parte de un fenómeno que resulta eminentemente complejo y que en el área de la Educación Física se ha caracterizado por evaluaciones de tipo estandarizadas como la aplicación de pruebas físicss que miden a todo(as) por igual, y con mediciones en el ámbito deportivo que privilegian el rendimiento y la técnica.

Categoría Previa Conocimiento del Contenido Específico - Categoría Primaria Rol del Profesor- Categoría Secundaria - Empatía Pedagógica.

En el marco de la categoría primaria Rol del Profesor, emerge la categoría secundaria empatía pedagógica, entendiendo que más que un instructor o un enseñante el profesor de Educación Física despliega capacidades humanas en la acción de educar.

«El rol en sí de profesor de Educación Física es un rol más cercano con los niños (...) más cariñoso con los chicos. Por ejemplo, la diferencia que van marcando... uno tiene que abrocharle los zapatos cuando son pequeños, tiene que acompañarlo si tiene alguna herida. Bueno, son cosas pequeñas pero que van fortaleciendo el lazo personal entre los alumnos y el profesor, se va rompiendo esa diferencia que había, entre los alumnos y el profesor». Entrevista Miguel [Cobertura 4,68\%]

El profesor de Educación Física tiene un rol algo diferente al profesor de aula. De acuerdo con el relato, está más cercano a los estudiantes, existe una mayor confianza con él, un mayor diálogo y comunicación, cuestión que probablemente tiene varias explicaciones como por ejemplo el espacio físico donde se realiza la clase, normalmente en el patio, en un gimnasio, en una cancha. Vale decir que los estudiantes se sienten en un espacio de mayor libertar para moverse, para correr, para jugar. Normalmente el profesor de Educación Física es una persona dinámica, con sentido lúdico, que realiza múltiples funciones al interior del establecimiento. Por lo general, él es quien está a cargo de las festividades de la escuela (aniversarios, desfiles, fiestas patrias, etc.), animando e invitando a participar a la comunidad en estas instancias. El currículo de la asignatura favorece el trabajo colaborativo y de equipo, con los deportes colectivos, o las cordadas en las salidas a terreno o senderismo. Por otro lado, en términos didácticos la disciplina promueve la inclusión, el respeto por el otro, y las actividades preferentemente lúdicas como juegos, circo, bailes, y manifestaciones de la cultura juvenil, expresiones conocidas como deportes urbanos tales como: slackline, acrossport, skate, parkour, escalada, y otros. Lo anterior identifica a los jóvenes con sus gustos e intereses, de ahí entonces que generar una relación de empatía a través de un estilo de enseñanza participativo favorecedor del diálogo y la interacción es fundamental para el profesor de Educación Física del siglo XXI.

Categoría Previa Conocimiento del Contenido Específico - Categoría Primaria Rol del Profesor- Categoría Secundaria- Motivación para la Clase

A partir del análisis de la categoría primaria Rol del Profesor, emerge como categoría secundaria motivación para la clase, bajo el principio que, para generar aprendizajes de calidad, es fundamental la motivación por parte de los estudiantes, vale decir que deseen participar de la actividad pedagógica.

«¿Cómo motivo al que llegó desmotivado?, ¿cómo le subo el ánimo? Va también por ese lado de tratar de cambiar el chip y que ellos al momento de empezar la clase de educación física, automáticamente se motiven y quieran trabajar». Entrevista Joaquín [Cobertura 3,34\%]

La motivación para la clase es una de las cuestiones más estudiadas y discutidas en la literatura pedagógica, toda vez que la motivación intrínseca es crucial para generar aprendizajes de calidad. Por otro lado, la falta de ella puede generar actitudes que obstaculizan el aprendizaje como la indisciplina, apatía y la resistencia pasiva (Vallejo, 2011). Al respecto, Ospina señala «cobra importancia también el papel del profesor, para establecer la relación adecuada entre la motivación y el aprendizaje en la construcción del conocimiento, dada su influencia decisiva en el desarrollo curricular» (2006, p.159). En este sentido el relato de los profesores de Educación Física adquiere un profundo sentido pedagógico, asumiendo la tarea de desarrollar acciones que motiven a sus estudiantes. Esto lo observamos cuando los profesores informantes describen como tarea, por ejemplo «subir el ánimo» o «cambiar el chip» de aquellos alumnos que llegan desanimados a las aulas, utilizando para ello distintas estrategias didácticas. Por otro lado, la utilización de estilos de enseñanza participativos, individualizadores, creativos y socializadores, vale decir preferentemente activos, son los que los profesores de EF tienden a implementar en sus aulas (Merino-Barrero, Valero-Valenzuela, \& Moreno-Murcia, 2017), cuestión que favorecerá la motivación y el deseo de participación por parte de los estudiantes.

Categoría Previa Conocimiento del Contenido Específico - Categoría Primaria Rol del Profesor- Categoría Secundaria - Profesor Lúdico.

La categoría hace referencia preferentemente a cómo el profesor aborda el proceso de enseñanza-aprendizaje.

«Aquí el profesor tiene que ser dinámico, si un profesor no es dinámico lamentablemente en educación física está ¡muerto!» Entrevista Juan [Cobertura 0,79\%]

En el relato de los profesores de Educación Física, se observa cómo estos valoran la actitud con la que el profesor inicia la sesión, señalando expresamente que una actitud lúdica puede influir positivamente en sus estudiantes, generando de esta manera una motivación extrínseca para con sus estudiantes y de esta manera hacerlos participes de la sesión. Con respecto a la noción de ludismo podemos comprenderla como una cualidad humana que permite gozar la vida, una sensación de satisfacción, de sentirse bien, de placer. En este sentido cada acto, actividad, pensamiento, 
instante de creación puede convertirse en un momento de diversión o esclavitud, cuestión que depende principalmente de la actitud con la que enfrentemos la vida. De ahí, entonces, que lo lúdico también es una decisión, lo construye cada persona, es como la energía que nos permite transformarnos y construirnos, y en ese ejercicio proyectar a las personas que nos rodean (Trigo, 2006; Trigo \& Montoya, 2007)

Categoría Previa Conocimiento del Contenido Específico - Categoría Primaria Rol del Profesor- Categoría Secundaria - Profesor Modelo

A partir del análisis de la categoría primaria Rol de Profesor, emerge como categoría secundaria profesor como modelo.

«Yo en general no soy tanto de demostrar lo que hay que hacer, pero lo hago, para que los niños en algún momento también vean que el profesor se maneja, sabe lo que está hablando, o sea, no es pura teoría, entonces, «puede hacerlo». Hay que retroalimentar esa parte porque los niños comúnmente dicen- «ningún profesor de educación física corre con uno, cuando hay que hacer abdominales o cuando hay que correr el profe está sentado o está por ahí haciendo nada». Entrevista Joaquín [Cobertura 1,87\%]

En términos generales, los sujetos investigados conciben al profesor de Educación Física como un modelo para sus estudiantes en al menos dos dimensiones. La primera dimensión daría cuenta de la demostración físico-técnica que hace el maestro de las diferentes ejercitaciones y/o movimientos referidos a los deportes y a la expresión motriz considerados en la matriz curricular del sector. Esta percepción evoca a un paradigma fuertemente instalado en el imaginario de la disciplina, en la que se entiende que la clase sería una sesión de acondicionamiento físico o deportes. Bajo esta mirada paradigmática el profesor debiera poseer un nivel elevado de cualidades físico-técnicas para demostrar y corregir aquellos movimientos y acciones erróneos ejecutadas por sus estudiantes. Lo anterior es más bien una mirada algo reduccionista de un fenómeno absolutamente complejo y que va tejiendo y enhebrando no sólo la dimensión físcomotriz, sino más bien es capaz de considerar los aspectos, emocionales, sociales, históricos, culturales y contextuales de la práctica física.

Categoría Previa Conocimiento Pedagógico General - Categoría Primaria Déficits en la Formación- Categoría Secundaria - En lo Administrativo

Los profesores entrevistados consideran importante los aspectos administrativos de la tarea docente.

«Yo creo que estamos más descendidos en la parte administrativa. La parte administrativa es nuestra debilidad y yo personalmente también me siento así. Siento que si me preguntan cuáles son mis falencias van por la parte administrativa». Entrevista Joaquín [Cobertura 1,49\%]

Los profesores entrevistados relatan que una de la mayor debilidad en su paso por la Universidad, durante el proceso de formación inicial, es la escasa preparación que tuvieron en los aspectos administrativos de la tarea docente, cuestión que los lleva a cometer algunos errores, generando dudas e incertidumbre en los primeros años de docencia. Esto se podría explicar con el hecho que los currículos formativos en la disciplinan privilegian los aspectos específicos del contenido, vale decir el área biológica, el área deportiva, el área del desarrollo motor y la expresividad, y el área de las actividades en contacto con la naturaleza. Por el lado de las practicas progresivas, los profesionales egresados valoran muy positivamente la instancia, no obstante, aparentemente no abordan esta tarea, pues los programas estarían orientados al desarrollo del ethos docente, al desarrollo de la vocación pedagógica, y en definitiva a la consolidación del saber pedagógico por parte del profesorado novel (Almonacid-Fierro, Merellano-Navarro, \& Moreno-Doña, 2014).

Categoría Previa Conocimiento Pedagógico General - Categoría Primaria Déficits en la Formación- Categoría Secundaria - Área Pedagógica.

«Sí, a lo mejor, quedo un poco al debe con lo que es trabajar con alumnos conflictivos, ¿cómo levantar más la motivación de los niños?, ¿cómo trabajar con niños que son un poco más inquietos o trabajar con niños que..., no sé, tengan asperger, autismo o alguna discapacidad motriz en ese sentido?» Entrevista Juan [Cobertura 1,17\%]

Los informantes señalan debilidades en el área de la formación pedagógica, entendiendo que este dominio es distinto al área disciplinar. Esta última comprendería los aspectos referidos a la atención a la diversidad, la utilización del espacio y los materiales deportivos, el rol de profesor jefe y el área de la orientación educativa. Con respecto a la atención a la diversidad, si bien es cierto las instituciones de Educación Superior han incorporado cursos para abordar las necesidades educativas especiales, en el relato se indica que los contenidos ahí desarrollados fueron vistos de forma superficial. Dicha situación generaría miedos e inseguridades al momento de vincular los aprendizajes con la realidad escolar.

Con respecto a los déficits pedagógicos que representa enseñar en condiciones ideales un contenido por ejemplo el baloncesto, con gimnasio y un balón para cada profesor en formación y seguidamente llegar a la escuela y encontrarse con un balón para 30 o 40 estudiantes, en un patio de tierra que con suerte puede contar con unigoles (Aro transportable de baloncesto sin tablero utilizado para practicar el tiro), genera una suerte de divorcio de la Formación Inicial Docente (FID), con la realidad escolar, cuestión que obviamente repercute en la motivación del propio docente. Por otro lado, la labor de orientación y asumir la tarea de profesor jefe (profesor responsable en lo académico-administrativo de un curso), son cuestiones que no aborda con suficiente potencia la FID, y más bien son tareas que se dejan a los primeros años de inclusión por parte del profesor en el sistema escolar, y que debe asumir la mayoría de las veces solo, sin un acompañamiento especializado, como podría ser un programa de mentoría, con un profesor experto que haga las veces de tutor del profesor novel por un periodo de uno o dos años.

Categoría Previa Conocimiento Pedagógico General - Categoría Primaria Pedagogía del Profesor- Categoría Secundaria - Actitud Indagativa

Esta categoría posee un porcentaje de frecuencia de 2,78 \% de la totalidad de la muestra de profesores de Educación 
Física investigados y un 5,7 \% de la categoría previa. Los profesores investigados reconocen que, como parte del conocimiento pedagógico del profesor, debe existir una actitud indagativa.

«Si algo no funcionó hay que ir a otra cosa, todos los días estar cambiando, que sale una nueva metodología, que hay una persona que está estudiando cierta parte, ahora estamos con el tema de la neurociencia, el estudio del cerebro. Hay que tener conocimiento de cómo reacciona el cerebro a diferentes estímulos. Entonces eso ya lo están metiendo en la educación y si uno no busca, no investiga se va quedando en el pasado, va quedando obsoleto y hay que estar en eso, no nos queda otra a los profes de educación física». Entrevista Joaquín [Cobertura 1,95\%]

Esta categoría secundaria da cuenta de cómo los profesores de Educación Física asumen los nuevos desafíos de la profesión docente, entendiendo que el conocimiento crece de manera exponencial y que en consecuencia la FID se transforma sólo en una formación de base. En este sentido, el profesor comprende que su conocimiento debe ser actualizado permanentemente, en función de un aprendizaje socialmente relevante, culturalmente pertinente y personalmente significativo, vale decir que haga sentido para él y para sus estudiantes.

Categoría Previa Conocimiento Pedagógico General - Categoría Primaria Pedagogía del Profesor- Categoría Secundaria - Actitudes Transversales

Esta categoría posee un porcentaje de frecuencia de 5,56 $\%$ de la totalidad de la muestra de profesores de Educación Física investigados y un 11,4 \% de la categoría previa.

«Tenemos que desarrollar habilidades blandas como el respeto, el trabajo en equipo... la organización, el liderazgo, todo ese tipo de cosas, etc.» Entrevista Manuel [Cobertura 0,80\%]

Esta categoría secundaria da cuenta de las competencias humanas que deben trabajar los profesores en su acción pedagógica, para superar la lógica individualista, la competencia y el exitismo. La escuela en consecuencia se transforma en el espacio social en que niños y jóvenes pueden vivenciar relaciones en las que el respeto, la empatía y la honestidad, por ejemplo, son los pilares en los que se funda y sustenta el proceso de aprendizaje. El profesor se convierte en una figura privilegiada y modelo a seguir en muchas ocasiones. Por lo tanto, el docente apunta a desplegar su saber pedagógico desde una perspectiva axiológica privilegiando el trabajo en equipo, la sana convivencia, el respeto, la capacidad de escuchar y comprender al otro.

Categoría Previa Conocimiento Pedagógico General - Categoría Primaria Planificación de la Enseñanza Categoría Secundaria - Planificación Contextualizada.

Esta categoría posee un porcentaje de frecuencia de 5,56 \% de la totalidad de la muestra de profesores de Educación Física investigados y un 11,4 \% de la categoría previa. Los profesores investigados exponen en sus relatos que el proceso de planificación de clases debe adecuarse al contexto donde se desarrolla la sesión de aprendizaje.

«No, eso es absolutamente dinámico no puede ser rígido, yo, si bien es cierto, preparo la clase, hago la planifica- ción, y para eso es fundamental conocer el curso. No es llegar y buscar, «ya tengo cuatro juegos y esto lo hago». Tengo que conocer al curso, porque obviamente hay cosas que no resultan en un curso y sí resultan en otro». Entrevista Joaquín [Cobertura 1,26\%]

En el relato de los profesores entrevistados la planificación cumple un papel importante, en cuanto permite la preparación para la enseñanza, la comprensión del contexto en términos de infraestructura, equipamiento, número de estudiantes, género, edad, motivación por el aprendizaje, etc. En este contexto, es el docente quien debe contextualizar los contenidos al aula y a las condiciones que allí se presentan, y en este sentido mientras mayores experiencias pedagógicas haya experimentado el docente en su vida laboral, mayores habilidades posee para adecuar los contenidos de enseñanza a la clase que le corresponde dictar en un momento determinado.

Categoría Previa Conocimiento Pedagógico General - Categoría Primaria Planificación de la Enseñanza Categoría Secundaria - Planificación Flexible

Esta categoría posee un porcentaje de frecuencia de 8,33 \% de la totalidad de la muestra de profesores de Educación Física investigados y un 17,1 \% de la categoría previa. Los profesores investigados reconocen que, como parte del conocimiento pedagógico general, es menester considerar que la planificación de clases es flexible.

«La planificación de la clase, o sea, si bien es cierto uno dentro la clase puede ir viendo aspectos y cambiando cosas e improvisando detalles, uno debe tener una planificación bien efectiva». Entrevista Joaquín [Cobertura 3,56\%]

En los relatos de los profesores entrevistados, se observa que ellos valoran contar con una planificación de la sesión, no obstante, su planteamiento es que debe tener un carácter flexible, pues son múltiples las variables que intervienen en la realización de una clase. Ahora bien, la relevancia de contar con una planificación bien estructurada y en el marco de las bases curriculares, dice relación con garantizar una enseñanza de calidad. La preparación de la clase constituye un medio para anticipar posibles dificultades, no obstante, su propósito es mantener un hilo conductor con respecto a los aprendizajes esperados, el desarrollo de los contenidos y el proceso evaluativo, habida cuenta que la clase propiamente tal constituye un hecho único e irrepetible. En este sentido, la sesión de Educación Física bien podría ser considerada un caso clínico, toda vez que las condiciones climáticas, los aprendizajes, las características de los estudiantes, el horario, los materiales y el ambiente en general, hacen precisamente que cada lección de clases sea única.

Categoría Previa Conocimiento Pedagógico General - Categoría Primaria Reflexión Sobre la Práctica- Categoría Secundaria - Instancia Reflexiva.

Esta categoría posee un porcentaje de frecuencia de 5,56 \% de la totalidad de la muestra de profesores de Educación Física investigados y un 11,4 \% de la categoría previa. Los profesores investigados plantean que la instancia reflexiva es un momento relevante para el ejercicio docente.

«La reflexión, yo creo que las primordiales se deben hacer en dos instancias, una clase a clase, en cada sesión y 
la otra la reflexión en la parte evaluativa (en las evaluaciones) si realmente estoy obteniendo lo que quiero de una evaluación o estoy evaluando para colocar la nota y sería todo. Uno constantemente tiene que estar reflexionando sobre ese tipo de evaluaciones, es algo que no se puede dejar, así como a la ligera». Entrevista Juan [Cobertura 1,64\%]

La instancia reflexiva, es una categoría que, desde la dimensión teórica y práctica, se puede comprender como un «saber que se recupera o construye desde la conciencia, en los continuos procesos de análisis crítico de la realidad escolar que el docente desarrolla de manera sistemática, a partir de la realidad del sistema escolar y las prácticas pedagógicas asociadas» (Almonacid-Fierro et al., 2014). Por su parte, los profesores entrevistados reconocen que los momentos reflexivos le permiten un proceso de autoevaluación de su práctica pedagógica. En este sentido los profesores comprenden que la revisión y análisis de su propia práctica es un ejercicio necesario para promover el aprendizaje con sus estudiantes, teniendo en cuenta que hay actividades que resultan y otras que no. Seguidamente estiman necesario evaluar los propios procesos metaevaluativos.

Categoría Previa Conocimiento Pedagógico General - Categoría Primaria Reflexión Sobre la Práctica- Categoría Secundaria - Retroalimentar para Aprender

Esta categoría posee un porcentaje de frecuencia de 4,17 \% de la totalidad de la muestra de profesores de Educación Física investigados y un 8,6 \% de la categoría previa. Los profesores entrevistados rescatan la posibilidad de ir retroalimentando el proceso formativo en diferentes instancias.

«La experiencia uno lo va teniendo clase a clase en la metodología, lo que va a funcionar, los juegos que funcionan los dejamos, los juegos que no funcionan les damos una vuelta, los retroalimentamos los vamos modificando para que vayan funcionando». Entrevista Silvio - [Cobertura 2,22\%]

En el análisis del discurso de los docentes informantes, se puede observar que conciben la retroalimentación como una instancia que permite la mejora de los procesos educativos, y este sentido se puede señalar que es la propia reflexión en la práctica la que permite generar más y mejores aprendizajes. Lo anterior puede entenderse desde las actividades prácticas que desarrolla el maestro, puesto que el docente piensa, idea y prueba nuevas acciones con las que se explora la situación problemática y con las que, al mismo tiempo verifica la comprensión de estas. Como producto de estas experiencias repetidas, comienza a construir conocimientos, estrategias, imágenes, expectativas y técnicas que le permitan valorar las situaciones a las que se enfrenta y decidir las acciones más adecuadas. Desde el punto de vista teórico se puede afirmar que el profesor tras reflexión en la acción puede realizar procesos de análisis sobre los procesos y resultados implicados en ella. El análisis no se centrará únicamente en las características de la situación o contexto del problema, sino que también se cuestionarán los procedimientos llevados a cabo para designar el problema y determinar su naturaleza, la formulación de objetivos, la selección de los cursos de acción y, sobre todo, las teorías implícitas y los modos de entender y de representar la realidad durante la acción.

\section{Discusión y conclusiones}

La investigación permitió dar cuenta sobre la manera en que los profesores interpretan ciertos aspectos de su quehacer pedagógico, vinculando permanentemente su práctica en aula y sus concepciones con respecto al CDC. Desde su práctica pedagógica los profesores destacan los aspectos asociados a los contenidos de carácter disciplinar, vale decir juegos predeportivos, deportes, habilidades motrices básicas, psicomotricidad, y las actividades motrices en contacto con la naturaleza, y en menor medida los aspectos asociados a la actividad física y salud. Estos serían los contenidos que mayormente utilizan en sus clases, al amparo de las bases curriculares y la normativa ministerial vigente. Seguidamente, se destaca que, para poder enseñar estos contenidos, se requiere un cierto manejo de carácter pedagógico, donde la asignatura de psicología, por ejemplo, juega un papel fundamental, pues permite conocer y comprender los distintos niveles evolutivos y de desarrollo de los niños. En su ejercicio docente, los profesores utilizan diversas estrategias para favorecer los procesos de aprendizaje de sus estudiantes, y en este sentido evocan las diversas posibilidades didácticas que se deben desplegar para el aprendizaje de todos y todas, entendiendo que la escuela no es la instancia para potenciar exclusivamente la técnica de los deportes o el rendimiento motor, muy por el contrario, se privilegia la motivación por la clase con una didáctica más inclusiva y participativa. Desde la práctica pedagógica de los profesores, el juego cumple un papel fundamental al momento de desarrollar un contenido, puesto que el juego integra y dinamiza la sesión, permite la colaboración y el respecto entre los participantes, y genera múltiples beneficios de carácter fisiológico. En consecuencia, es un aspecto didáctico fundamental en el desarrollo de las sesiones de Educación Física.

En este contexto, el rol de profesor de Educación Física es fundamental en las distintas instancias de aprendizaje que genera con sus estudiantes, constituyéndose en un modelo a seguir y en una figura protagónica por su estrecho vínculo con los niños, que ven en el juego una posibilidad para expresar toda su corporeidad y emocionalidad. Por otro lado, los jóvenes se identificarían con las distintas expresiones de la cultura juvenil que tiene cabida en el aula de Educación Física.

En términos de conocimiento, parece ser de vital importancia, de acuerdo con el discurso del profesorado proveniente de su práctica de aula, permitir el espacio para un aprendizaje de carácter diferenciado, en que todos los niños y niñas tengan la posibilidad de ampliar su capital motriz, sin discriminar a los más hábiles de los menos hábiles, vale decir una Educación Física integradora. Luego se requeriría que el conocimiento que se comparte en el área se convierta en un aprendizaje significativo para los alumnos, que puedan visualizar con claridad los beneficios para su calidad de vida y salud, a través de acciones lúdico-motrices orientadas a la diversidad y utilizando diferentes estrategias didácticas. Entonces es posible afirmar que el CDC es una cuestión fundamental para el profesor al momento de impartir clases, al poner el énfasis no sólo en el contenido, sino más bien, como enseñar ese contenido.

Los contenidos mayormente enseñados y trabajados en 
el área de Educación Física son más bien los tradicionales, que provienen fundamentalmente de las bases curriculares aprobadas el año 2013, y de la normativa emanada desde el Ministerio de Educación. Los profesores por cuestiones de infraestructura, equipamiento e implementación recurren a los deportes más conocidos como el fútbol, el básquetbol y el voleibol, el desarrollo de las habilidades motrices básicas en la primera infancia, el acondicionamiento físico para la salud con escolares de segundo ciclo básico y de enseñanza media y las expresiones rítmicas se reducen a los bailes folclóricos nacionales. Por su parte las actividades en contacto con la naturaleza se vivencian escasamente con estudiantes preadolescentes y/o adolescentes, básicamente por la serie de restricciones administrativas que conllevan las salidas pedagógicas de este tipo. Similar fenómeno ocurre con las actividades de expresión corporal y las manifestaciones contemporáneas de la cultura juvenil, cuestión que conlleva a la desmotivación de los estudiantes de secundaria principalmente por la asignatura, y consecuentemente a estilos de vida poco saludables de la población en su conjunto.

En términos generales, los profesores señalan que cuentan con un adecuado dominio de los contenidos disciplinares, es decir, dominan el currículo del área planteado en las bases curriculares. No obstante, poseer el conocimiento de carácter disciplinar no garantiza un aprendizaje de calidad por parte de los estudiantes, puesto que para lograr aquello es menester generar un ambiente propicio que considere algunos aspectos claves, como la motivación por parte del profesor, y el despliegue de estrategias de enseñanza pertinentes y contextualizadas al grupo curso que se está formando. Lo anterior, da cuenta de que los profesores requieren un conocimiento pedagógico general, que les permite planear, organizar y ejecutar situaciones de enseñanza-aprendizaje con la idea de alcanzar objetivos más amplios que tienen que ver con la formación de los estudiantes.

La formación de profesores de Educación Física en Chile se ha caracterizado en las últimas décadas por transitar desde un modelo pedagógico sustentado en una racionalidad tecnológica y positivista, hacia modelos de formación amparados en perspectivas más bien constructivistas, no obstante, algo lejos de aquellos que pregonan por enfoques desde la teoría crítica del currículo y la enseñanza. En este sentido el vínculo entre la FID y la realidad de la escuela genera en la mayoría de las veces un cierto divorcio, que se acentúa en los déficits de carácter pedagógico con los que llegan los profesores al ejercicio de la docencia. Lo anterior, se manifiesta en un débil dominio de las aulas, fundamentalmente por su diversidad, y las características de la cultura juvenil y, por otro lado, porque en la escuela no existen las condiciones ideales de infraestructura e implementación para desarrollar los contenidos de carácter técnico-deportivo, lo que obliga a los profesores a buscar estrategias pedagógicas más inclusivas, pedagógicamente más pertinentes y bajo un paradigma de tipo constructivista, que es en definitiva el que requiere el sistema escolar.

\section{Referencias}

Almonacid-Fierro, A., Feu, S., \& Carrizosa, M. V. (2018). Vali- dación de un cuestionario para medir el Conocimiento Didáctico del Contenido en el profesorado de Educación Física. Retos: Nuevas tendencias en Educación Física, Deporte y Recreación(34), 132-137.

Almonacid-Fierro, A., Merellano-Navarro, E., \& MorenoDoña, A. (2014). Caracterización del saber pedagógico: Estudio en profesorado novel. Revista Electrónica Educare, 18(3).

Amade-Escot, C. (2000). The contribution of two research programs on teaching content:»Pedagogical content knowledge» and «didactics of physical education». Journal of Teaching in Physical Education, 20(1), 78101.

Barnett, J., \& Hodson, D. (2001). Pedagogical context knowledge: Toward a fuller understanding of what good science teachers know. Science Education, 85(4), 426453.

Benito-Capa, Á., Portela-Lozano, A., \& Rodríguez-Jiménez, R. (2006). Análisis de la enseñanza de la Física en Europa: el fomento de competencias generales en estudiantes universitarios. Revista Iberoamericana de educación, 38(7), 1-5.

Bolivar, A. (1993). Conocimiento didáctico del contenido y formación del profesorado: El programa de Lee Shulman. Revista Interuniversitaria de Formación del Profesorado, $16(1), 113-124$.

Canales, M. (2006). Metodologías de investigación social. Introducción a los oficios. Santiago.

Cañadas, L., Santos-Pastor, M., \& Castejón, F. (2019) Competencias docentes en la formación inicial del profesorado de educación física. Retos: Nuevas Tendencias en Educación Física, Deporte y Recreación, 35, 284-288

Capel, S., Hayes, S., Katene, W., \& Velija, P. (2009). The development of knowledge for teaching physical education in secondary schools over the course of a PGCE year. European Journal of Teacher Education, 32(1), 51-62.

Castejon, F., \& Giménez, F. (2017). Conocimiento del contenido y conocimiento pedagógico del contenido de educación física en educación secundaria. Retos: Nuevas tendencias en Educación Física, Deporte y Recreación, 32, 146-151.

Coffey, A., \& Atkinson, P. (2003). Encontrar el sentido a los datos cualitativos: estrategias complementarias de investigacion: Medellin: Editorial Universidad de Antioquia.

Delgado, J., \& Gutiérrez, J. (1999). Métodos y técnicas cualitativas de investigación en ciencias sociales. Madrid.

Fenstermacher, G. (1994). The Knower and the Know: The Nature of Knowledge in Research in Education. Review of Research in Education, 20, 3-56.

Fernandez, C. (2015). Revisitando a base de conhecimentos e o conhecimento pedagógico do conteúdo (PCK) de professores de Ciências. Ensaio Pesquisa em Educação em Ciências, 17(2), 500-528.

Flores, R. (2009). Observando observadores: una introducción a las técnicas cualitativas de investigación social. Santiago de Chile: Ediciones Universidad Católica de Chile.

García, H. (1997). La formación del profesorado de educa- 
ción física: problemas y expectativas (Vol. 48). Barcelona: INDE publicaciones.

Gibbs, G. (2012). El análisis de datos en investigación cualitativa. Madrid: Ediciones Morata.

Gitomer, D., \& Zisk, R. (2015). Knowing what teachers know. Review of Research in Education, 39(1), 1-53.

Glaser, B., \& Strauss, A. (2017). Discovery of grounded theory: Strategies for qualitative research: Routledge.

Hunuk, D., Ince, M. L., \& Tannehill, D. (2013). Developing teachers' health-related fitness knowledge through a community of practice: Impact on student learning. European Physical Education Review, 19(1), 3-20.

Jaramillo, L., Murcia, N., \& Portela, H. (2005). La educación física: ¿ un problema de preparación o seducción. Armenia: Universidad del Cauca.

Jimenez, J., Rossi, F., \& Gaitán, C. (2017). La práctica reflexiva como posibilidad de construcción de saberes. Aportes a la formación docente en Educación Física. Movimento, 23(2), 587-600.

Kirk, D. (2001). Fundamentos para una pedagogía crítica en la formación del profesorado de educación física. En $\mathrm{La}$ educación física, el deporte y la salud en el siglo XXI (pp. 101-110): Editorial Marfil.

Kvale, S. (2011). Las entrevistas en investigación cualitativa. Madrid: Ediciones Morata.

López, V. Monjas, R. Pérez, D. (2003). Buscando alternativas a la forma de entender y practicar la educación física escolar . Barcelona: INDE publicaciones.

MacPhail, A., Tannehill, D., \& Karp, G. (2013). Preparing physical education preservice teachers to design instructionally aligned lessons through constructivist pedagogical practices. Teaching Teacher Education, 33, 100-112.

Mancha, J. (2012). Formación inicial y conocimiento didáctico del contenido en los profesores de educación física de secundaria de la ciudad de Badajoz. (Doctorado ), Universidad de Extremadura, España,

Marcon, D., Graça, A., \& Nascimento, J. (2012). Analysis of the pedagogical knowledge of prospective physical education teachers. Kinesiology, 44(2), 113-122.

Marcon, D., Nascimento, J., \& Graça, A. (2007). A construção das competências pedagógicas através da prática como componente curricular na formação inicial em Educação Física. Revista Brasileira de Educação Física e Esporte, 21(1), 11-25.

Merino-Barrero, A., Valero-Valenzuela, A., \& Moreno-Murcia, J. (2017). Análisis psicométrico del cuestionario estilos de enseñanza en educación física. Revista Internacional de Medicina y Ciencias de la Actividad Fisica, 17(66), 225-241.

Ministerio de Educación de Chile. (2013). Bases Curriculares Educación Básica. Recuperado de Chile: www.mineduc.cl

Moreno, A., Campos, M., Almonacid, A., \& Vargas, A. (2013). La educación física chilena. Un modelo tecnocrático de la enseñanza y desvalorización del colectivo docente. Tandem. Didáctica de la Educación Física, 42, 7-17.

Nascimiento, J. (2006). Formação do profissional de educação física e as novas diretrizes curriculares: reflexões sobre a reestruturação curricular. Rio Claro:
Biblioética.

Ospina, J. (2006). La motivación, motor del aprendizaje. Revista Ciencias de la Salud, 4(2), 158-160.

Pedroso, P. (1996). Alternância, modelos de formação e culturas pedagógicas. Revista Inovacao, 20(9), 236-282.

Ramos, V., dos Santos Graça, A., \& do Nascimiento, J. (2008). O conhecimento pedagógico do conteúdo: estrutura e implicações à formação em educação física. Revista Brasileira de Educação Física e Esporte, 22(2), 161171.

Rodríguez, G, Gil, J., \& García, J. (1996). Metodología de la investigación cualitativa. Málaga: Ediciones Aljibe.

Schön, D. (1998). El profesional reflexivo: como piensan los profesionales cuando actúan: Barcelona: Paidós.

Shulman, L. (1986). Those who understand: Knowledge growth in teaching. Educational Researcher, 15(2), 414.

Shulman, L. (1987). Knowledge and teaching: Foundations of the new reform. Harvard Educational Review, 57(1), 1-23.

Stran, M., \& Curtner-Smith, M. (2010). Impact of different types of knowledge on two preservice teachers' ability to learn and deliver the Sport Education model. Physical Education Sport Pedagogy, 15(3), 243-256.

Strauss, A., \& Corbin, J. (2002). Bases de la investigación cualitativa: técnicas y procedimientos para desarrollar la teoría fundamentada. Medellín: Universidad de Antioquia.

Taylor, S., \& Bogdan, R. (1987). Introducción a los métodos cualitativos de investigación (Vol. 1), Barcelona: Paidós.

Trigo, E. (2006). Inteligencia creadora, ludismo y motricidad. Acción 3 Consentido. Popayan: Universidad del Cauca.

Trigo, E., \& Montoya, H. (2007). Aportes de la motricidad humana a la Educación Física. Motricidad y Persona, 3, 9-43.

Vallejo, G. (2011). El aprendizaje desde la perspectiva de la motivación profesional y las actitudes. Medisan, 15(3), 363-368.

Vázquez-Bernal, B., \& Jiménez-Pérez, R. (2019). El conocimiento didáctico del contenido (CDC) de una profesora de ciencias: reflexión y acción como facilitadores del aprendizaje. Enseñanza de las Ciencias, 37(1), 25-53. doi:10.5565/rev/ensciencias.2550

Ward, P., Kim, I., Ko, B., \& Li, W. (2015). Effects of improving teachers' content knowledge on teaching and student learning in physical education. Research Quarterly for Exercise and Sport, 86(2), 130-139.

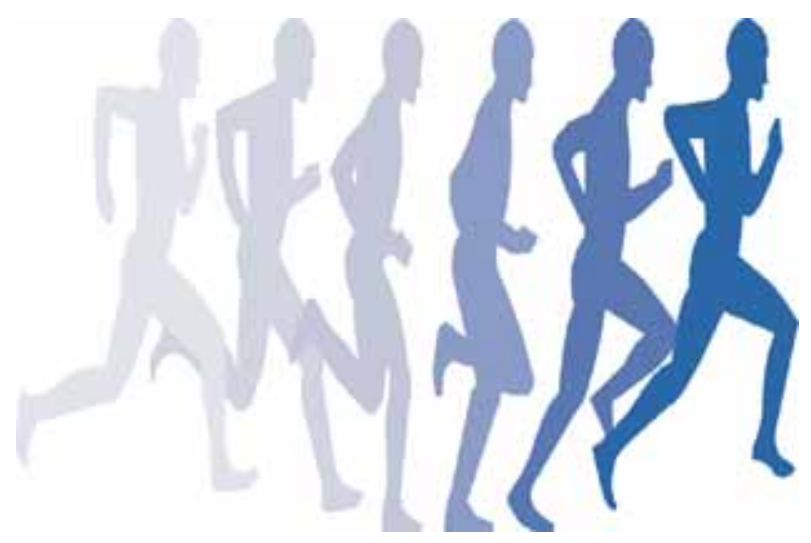

